\title{
Changes in corticomotor excitability following prolonged muscle tendon vibration
}

\author{
Arturo Forner-Cordero ${ }^{\mathrm{a}, \mathrm{b}, *}$, Maarten Steyvers ${ }^{\mathrm{a}}$, Oron Levin ${ }^{\mathrm{a}}$, \\ Kaat Alaerts ${ }^{\text {a }}$, Stephan P. Swinnen ${ }^{\text {a }}$ \\ ${ }^{a}$ Motor Control Laboratory, Department of Biomedical Kinesiology, Group Biomedical Sciences, Katholieke Universiteit Leuven, Belgium \\ ${ }^{\mathrm{b}}$ Instituto de Automática Industrial (IAI-CSIC), Ctra de Campo Real km. 0200, 28500 Arganda del Rey, Madrid, Spain
}

Received 20 June 2007; received in revised form 13 January 2008; accepted 4 February 2008

Available online 20 February 2008

\begin{abstract}
The present experiment addressed whether increases in corticospinal excitability following sensory stimulation with muscle tendon vibration are accompanied by reorganization of the forearm musculature representation within the primary motor cortex. Using transcranial magnetic stimulation, we mapped the corticomotor projection to the dominant flexor carpi radialis (FCR) and extensor carpi radialis brevis (ECR) muscle before and after interventional sensory stimulation obtained via muscle tendon vibration ( $80 \mathrm{~Hz}, 60 \mathrm{~min})$ to the dominant distal wrist flexor tendons. Following vibration, MEP amplitude at the optimal stimulation position, motor output area, as well as map volume, increased significantly for the ECR. None of these effects reached significance for the FCR. These results suggest that the antagonistic vibratory response (AVR), which is considered to be of cortical origin, induces a delayed facilitation of musculature that is antagonistic to the site of the directly activated Ia afferent pathways. This example demonstrates that peripheral sensory stimulation can induce lasting increases in corticospinal excitability in the absence of actual movements.

(C) 2008 Elsevier B.V. All rights reserved.
\end{abstract}

Keywords: Muscle tendon vibration; Somatosensory stimulation; Transcranial magnetic stimulation; Mapping; Plasticity

\section{Introduction}

The adult human sensory and motor cortices have the potential to reorganize rapidly in response to changing environmental conditions (for a review, see references $[3,14]$ ). The term 'neuroplasticity', defined as 'any enduring changes in cortical properties like strength of internal connections, representational patterns, or neuronal properties, either morphological or functional', refers to this ability [14]. Experimental interventions and pathological conditions that have shown to induce reorganization changes in motor cortical representations are: immobilization [25], ischemic nerve block [1,2,69], motor learning and practice of relatively unskilled movements $[11,19,20,21,34,35]$, amputation $[9,12,16]$, spinal cord injury $[24,59]$ and stroke $[10,27,60]$.

\footnotetext{
* Corresponding author at: Instituto de Automática Industrial (IAI-CSIC), Ctra de Campo Real km. 0200, 28500 Arganda del Rey, Madrid, Spain. Tel.: +34 9187119 00; fax: +34 918717050 .

E-mail address: aforner@iai.csic.es (A. Forner-Cordero).
}

The potential role of somatosensory stimulation as a means to dynamically modulate the organization of the motor cortex has been postulated during the past years $[1,2,48,52]$. Hamdy et al. [18] were the first to demonstrate that even a relatively short period $(10 \mathrm{~min})$ of electrical pharyngeal stimulation is sufficient to trigger changes in the pharynx' M1 representation in neurologically intact individuals. Ridding et al. [41,42] reported similar findings following interventional electrical peripheral nerve stimulation over longer periods of time. Further exploration has strengthened the understanding that electrical peripheral nerve stimulation is a powerful tool to induce sustained excitability increases as well as rapidly evolving neuroplastic changes of the human motor cortex $[8,28,29]$. Peripheral electrical nerve stimulation has also been shown to drive recovery of motor functions following upper motor lesions, e.g., improving swallowing functions in individuals with stroke [15].

Passive movement is also commonly applied as a proprioceptive stimulation technique in neurorehabilitation. This form of somatosensory stimulation has been found to generate brain activity not only in sensory, but also in motor cortical areas in humans $[7,39,64]$ and/or induce persistent neuroplastic changes 
of the sensorimotor representation when administered repeatedly [7].

Muscle tendon vibration has also shown to elicit motor cortex activation [31,32]. Recent studies with TMS have demonstrated its direct modulating effect on M1 excitability with a vibration intervention below the sensory illusion threshold $[45,46]$. Yet a comparative analysis of brain activity with positron emission tomography showed that both the loci and levels of activation during tendon vibration did not match to those obtained during passive movement [39], albeit both techniques predominantly activated the proprioceptive Ia-afferents pathways [43,44]. However, when the tendon vibration was accompanied by sensory illusion, cortical activation levels surpassed those measured with tendon vibration alone [39]. Given the dynamic nature of motor cortex reorganization [11,51] and its critical dependence on afferent stimulation parameters $[37,51,68]$, the quest for rationally founded treatment procedures that produce the most beneficial therapeutically relevant plasticity, is open.

In humans, representational cortical plasticity can be assessed at a regional level by means of transcranial magnetic stimulation (TMS) mapping of corticomotor representations [50,56,61,66]. The TMS mapping technique has been used extensively to address dynamic changes in corticomotor representations following various experimental and pathological conditions, like brain or spinal cord injury $[2,13,15,35,59]$. To evaluate the maps, single TMS pulses are delivered via a focal figure-of-eight coil to scalp positions arranged in a coordinate system overlying the primary motor cortex (M1). By measuring the motor evoked potential (MEP) amplitude in the targeted muscle(s), 'maps' based upon spatial changes in MEP amplitude among multiple stimulation positions can be composed. In this way, a functional topographic map of the M1 projection to hand and forearm muscles can be obtained. Motor output maps can be quantified by a number of variables, such as the optimal stimulation position, the map area and volume, and the center of gravity (CoG) $[61,66]$.

In a previous study, we reported that a $30 \mathrm{~min}$ period of muscle tendon vibration to the wrist flexors, is sufficient to induce a lasting (up to 30-60 min after the end of vibration) corticospinal excitability increase in the antagonistic wrist extensor muscles [53]. When tendon vibration provokes a sensory illusion, it is accompanied by an antagonistic vibratory response (AVR) that is reflected in increased EMG activity of the antagonist muscle, similar to voluntary motion [5]. Previous evidence has suggested that the AVR may result from a perceptual-to-motor transformation of proprioceptive information, rather than from spinal reflex mechanisms [5]. This intervention is possibly relevant in the search for protocols that promote functional recovery after central nervous system injury [13].

To gain further insight into the impact of interventional muscle tendon vibration on corticomotor excitability [22,45,54], we investigated by means of TMS mapping whether these excitability changes are accompanied by a reorganization in M1. Previous studies have demonstrated that the TMS mapping technique is sensitive to detect changes in the motor representation, following somatosensory stimulation paradigms $[28,29,41]$. Consequently, we wondered whether a recently introduced type of interventional somatosensory stimulation, i.e., muscle tendon vibration, has the potential to drive changes in human motor cortex organization.

\section{Materials and methods}

\subsection{Subjects}

Eleven neurologically healthy right-handed [33] volunteers participated in the present study (six males, five females, mean age \pm S.D.: $23 \pm 6.14$ years). The participants were naive about the purpose of the experiment, were screened for potential risk of adverse events during TMS [63], and provided written informed consent prior to participation. The experimental procedures were approved by the local Ethics Committee for Biomedical Research at the Katholieke Universiteit Leuven, according to the Declaration of Helsinki.

\subsection{Experimental set-up}

Subjects were seated comfortably in front of a manipulandum that stabilized their right forearm in a horizontal semi-prone position. A cushioned support fixated the subject's forearm, and the hand was inserted in a hand-piece with the wrist secured in a neutral posture. An electromagnetic mechanical stimulator (Tiravib 50020, Tira GmBH, Schalkau, Germany) was mounted on the manipulandum, with the vibrator probe pressing perpendicularly to the distal tendons of the wrist flexor muscles under an average load of 15.64 N (Kulite ECD-500N) (Fig. 1).

\subsection{Electromyographic recordings}

Surface electromyographic (EMG) activity was recorded from the right m.flexor carpi radialis (FCR) and m.extensor carpi radialis brevis (ECR) by means of disposable disc electrodes. Electrodes were placed $2 \mathrm{~cm}$ apart over the middle portion of the muscle belly, and aligned longitudinally with the muscle (Fig. 1). The pre-amplified signal was amplified $(\times 1000)$ and bandpass-filtered $(15-1000 \mathrm{~Hz})$ (Noraxon Myosystem 2000), prior to sampling at $5 \mathrm{kHz}$ using a CED Power 1401 analogue-digital converter (Cambridge Electronic Design, Cambridge, UK). Data were stored on a personal computer for off-line analysis (Signal software; Cambridge Electronic Design).

\subsection{Transcranial magnetic stimulation}

Single-pulse transcranial magnetic stimuli were delivered by means of a Dantec MagLite r-25 stimulator (Medtronic, Skovlunde, Denmark) (maximal stimulator output: $1.5 \mathrm{~T}$ ) with a figure-of-eight coil (MC-B70 magnetic coil transducer, outer radius diameter: $50 \mathrm{~mm}$ ). The magnetic stimulus had a bipha-

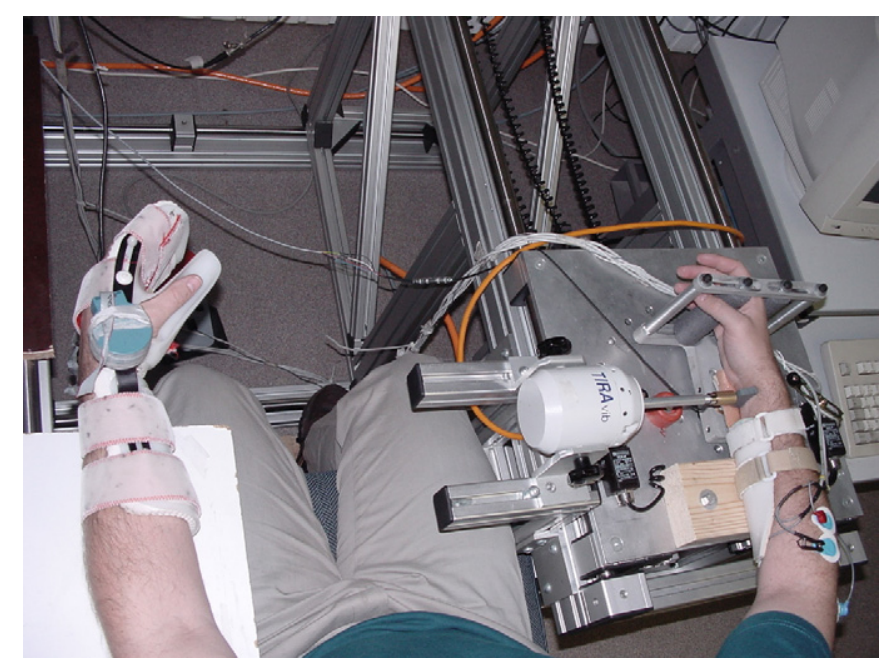

Fig. 1. View of the experimental set-up. 


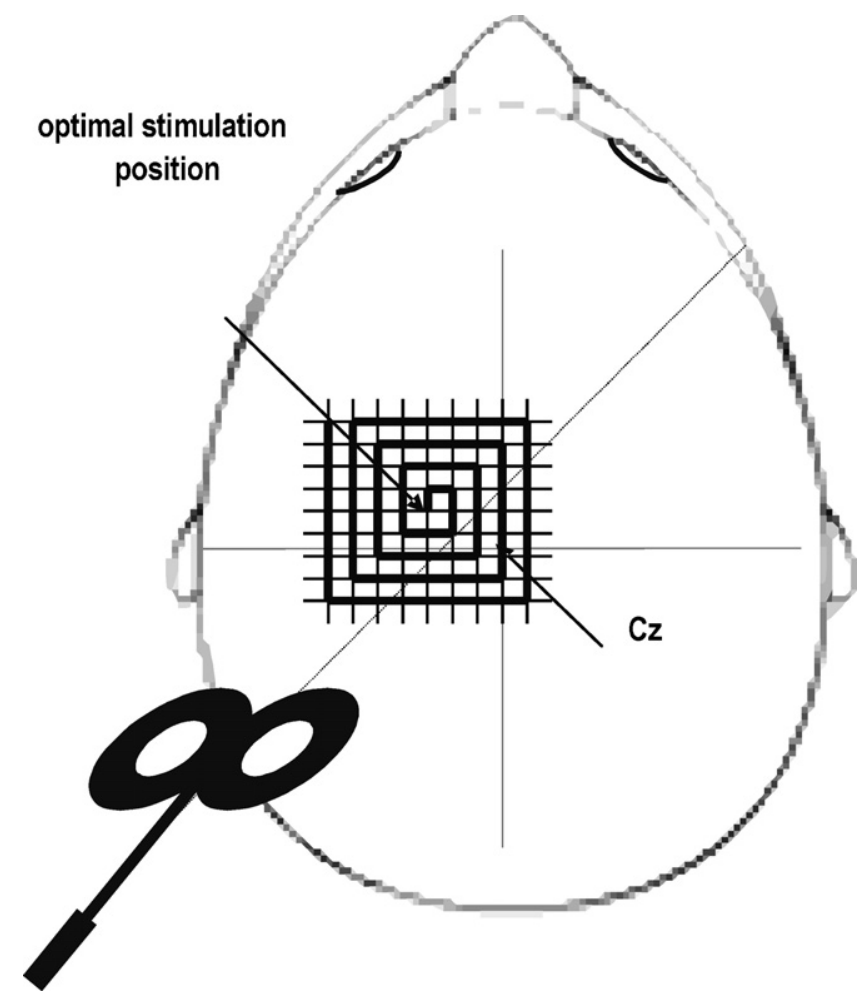

Fig. 2. Illustration of the TMS mapping procedure.

sic pulse configuration with a pulse width of $280 \mu$ s. The coil was positioned tangentially to the scalp over the subjects' left hemisphere with the coil handle pointing backward and rotated $45^{\circ}$ away from the midsagittal line [65]. The optimal stimulation position for eliciting MEPs in the right FCR was marked with a soft-tip pen. In all cases, a response of the ECR was also evoked in this position. Stimulation intensity for mapping of the FCR- and ECR M1 representation was initially set at $120 \%$ of the FCR rest motor threshold (rMT). rMT was determined at the optimal stimulation position as the lowest intensity needed to evoke MEPs in the relaxed FCR of at least $50 \mu \mathrm{V}$ amplitude in 5 out of 10 consecutive trials [47]. If needed, stimulation intensity was additionally adjusted to obtain test MEP amplitudes between 200 and $400 \mu \mathrm{V}$ for the FCR at rest at the optimal stimulation position.

\subsection{Corticomotor mapping}

The FCR- and ECR representation areas were mapped with a protocol modified from [66]. Subjects wore a tight-fitting cap with a $1-\mathrm{cm} \times 1-\mathrm{cm}$ orthogonal coordinate system referenced to the vertex $(\mathrm{Cz})$. The cap was positioned using cranial landmarks (nasion-inion) and the pre-auricular creases as references. Single TMS pulses (interstimulus interval: $6 \mathrm{~s}$ ) were applied in $1 \mathrm{~cm}$-steps in a clockwise spiral course beginning at the optimal stimulation position for the FCR [28] (Fig. 2). Data collection was initiated $50 \mathrm{~ms}$ prior to the delivery of TMS and lasted $150 \mathrm{~ms}$. Each stimulation position was stimulated two times (one time in one subject) before moving to the adjacent grid point, until the border of the motor maps of both target muscles had been defined. This procedure was repeated three times (six times in one subject) both before and immediately following interventional vibration, resulting in a total of six stimuli per stimulation site for both conditions. It was previously demonstrated that three stimuli per condition is sufficient to produce reliable and reproducible maps [30]. Each mapping procedure had a duration of $30-60 \mathrm{~min}$.

\subsection{Interventional muscle tendon vibration}

An interventional protocol was employed, consisting of wrist flexor tendon vibration for a total of $60 \mathrm{~min}(80 \mathrm{~Hz}$, cycle duration $30 \mathrm{~s}: 25 \mathrm{~s}$ on- $5 \mathrm{~s}$ off $)$. Vibration amplitude was adjusted prior to the start of the intervention to induce optimal kinaesthetic illusions at $80 \mathrm{~Hz}$ [17], and was kept constant throughout the experiment. The vibrator was driven by a digital output signal generated by means of a Power 1401 system (Cambridge Electronic Design). A similar vibration protocol, applied for $30 \mathrm{~min}$, resulted in a lasting facilitation for the antagonistic ECR [53]. The motor output area of the FCR and ECR was mapped prior to and immediately following the vibration session. During vibration, vision was prevented by a blindfold and subjects were instructed to relax their forearm muscles as much as possible, to focus their attention to the vibrated wrist, and not to suppress the occurrence of any kinaesthetic illusion. FCR and ECR EMG activity were monitored and recorded throughout vibration.

In order to evaluate the elicited kinesthetic illusions, subjects were instructed to match the direction and amplitude of the vibration-induced illusory movement on the right wrist by means of moving their left wrist mirror-wise (e.g., CalvinFiguiere et al. $[5,6])$. A wrist-hand orthosis was mounted on the left forearm and hand. This orthosis was used to restrict the wrist motion to flexo-extension and to measure the wrist angle (Fig. 1). The frictionless axis of the orthosis was aligned with the anatomical axis of the wrist joint. Angular displacements were registered by means of a high-precision shaft encoder fixed to the movement axis of the orthosis (HP, 8192 pulses/rev), digitized $(100 \mathrm{~Hz})$ and saved on a PC for off-line analysis. This procedure was repeated four times, every $15 \mathrm{~min}$ from the beginning of the vibration intervention, to control for the effectiveness and stability of Ia-afferent stimulation throughout the intervention. Each measurement period consisted of five cycles wrist flexor vibration ( 1 cycle: $25 \mathrm{~s}$ on- $5 \mathrm{~s}$ off).

\subsection{Data analysis}

The size of the FCR and ECR MEPs was measured offline by calculating the peak-to-peak amplitude of each waveform within a time window from 10 to $40 \mathrm{~ms}$ following the delivery of each magnetic pulse. The motor representation area of the FCR and ECR was defined as the number of stimulus positions whose stimulation evoked a mean MEP in the target muscle with a peak-to-peak amplitude of at least $100 \mu \mathrm{V}$ (='active' stimulation positions). The peak-topeak amplitudes of the FCR and ECR MEPs obtained at the same stimulation site were averaged. Mean peak-to-peak amplitudes of MEPs obtained at each scalp site were plotted against anteroposterior and mediolateral distance. 3Dplots of mean motor output areas for both target muscles were composed by linear interpolation of the mean MEP-amplitudes between adjacent stimulation positions (Matlab 6, MathWorks, Inc.). Map volume referred to the sum of the mean amplitudes at all active stimulation positions for FCR and ECR separately. The center of gravity $(\mathrm{CoG})$ was computed separately for the FCR and ECR as a measure of the amplitude-weighted centre of the motor representational map $[26,61]$. It is expressed as a bivariate measurement with a medio-lateral $(x)$ and rostro-caudal coordinate $(y)$, using the following formula: $\operatorname{CoG}=\left[\sum a_{i} x_{i} / \sum a_{i}\right.$, $\sum a_{i} y_{i} / \sum a_{i}$ ], for stimulation position coordinates $x_{i}, y_{i}$ and amplitudes $a_{i}$. The magnitude of the CoG displacement vector, this is the Euclidean distance between the $\mathrm{CoG}$ before and after interventional muscle tendon vibration, was calculated [42].

Mean rectified EMG of the FCR and ECR was measured in a time window from 40 to $10 \mathrm{~ms}$ prior to the delivery of each magnetic stimulus to control for EMG silence at the moment of TMS. Additionally, mean rectified FCR and ECR EMG was calculated during the interventional protocol in order to check for changes in background EMG during vibration.

\subsection{Statistics}

Mapping variables before and after vibration were statistically compared by means of advanced linear models applications (STATISTICA 6.0, StatSoft Inc.). It was first tested whether the two wrist muscles responded similarly to the stimulation by using a one-way ANOVA with muscle (two levels) as factor. To investigate a possible temporal evolution of the mapping variables following the end of vibration, the first two $\left(\mathrm{POST}^{1,2}\right)$ and the last two $\left(\mathrm{POST}^{2,3}\right)$ spirals were grouped and separately compared to the PRE-values for each muscle separately using a one-way ANOVA with Time (three levels) as factor.

The spirals were combined in order to obtain averages of at least six MEPs at each stimulation site. Therefore, $\mathrm{POST}^{1,2}$ represents the average of the two first spirals, corresponding to the first $30 \mathrm{~min}$ after the intervention, while $\mathrm{POST}^{2,3}$ consists of the average of the last two spirals, i.e., $15-45$ min post-intervention. 
Additionally, in order to obtain a measure of CoG stability, the first three and last three MEPs sampled at each stimulation position during the PRE-mapping, were averaged into two separate maps $\left(\mathrm{PRE}^{\mathrm{A}}\right.$ and $\mathrm{PRE}^{\mathrm{B}}$, respectively) and statistically compared between each other and with the post-vibration measurements.

Mean amplitude of illusory movement was analyzed by means of a oneway (Time) repeated measures ANOVA. Time referred to the four periods of amplitude matching throughout the vibration intervention: at the start of vibration (Time 1), after $15 \mathrm{~min}$ (Time 2), $30 \mathrm{~min}$ (Time 3 ) and $45 \mathrm{~min}$ (Time 4) of vibration.

Finally, mean rectified EMG values of the FCR and ECR were analyzed by means of a one-way (condition) repeated measures ANOVA. The factor condi- tion consisted of three levels: pre-vibration, vibration and post-vibration. When significant effects were found, post hoc Tukey testing was conducted to identify the loci of these effects. The level of significance was set at $p<0.05$.

\section{Results}

The rest motor threshold rMT (mean \pm S.D.) of the FCR was $33.7 \pm 6.5 \%$ of maximal stimulator output. Actual stimulation intensity was set at $39.7 \pm 8.4 \%$ of maximal stimulator output.
FCR

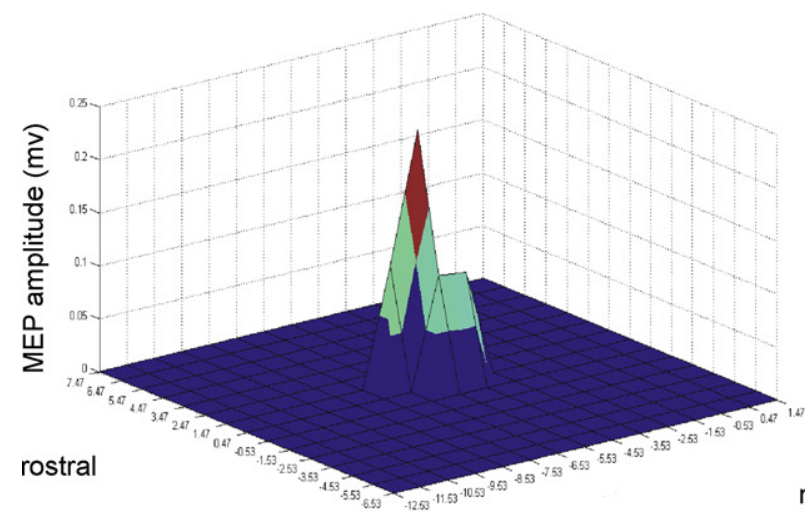

ECR

PRE

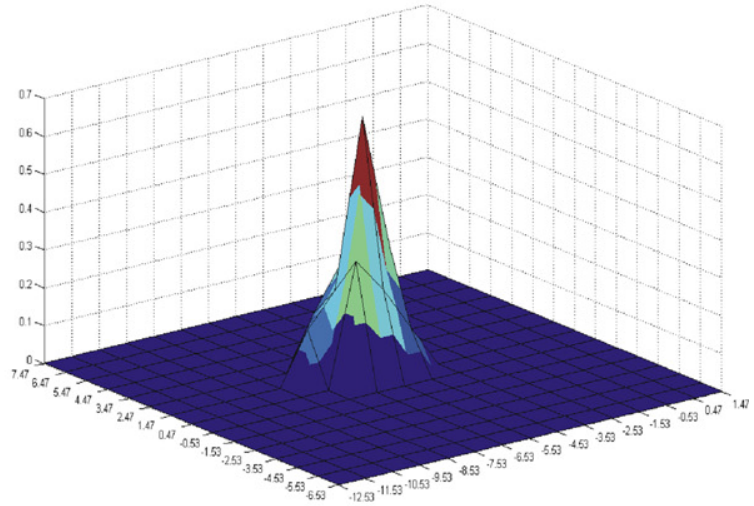

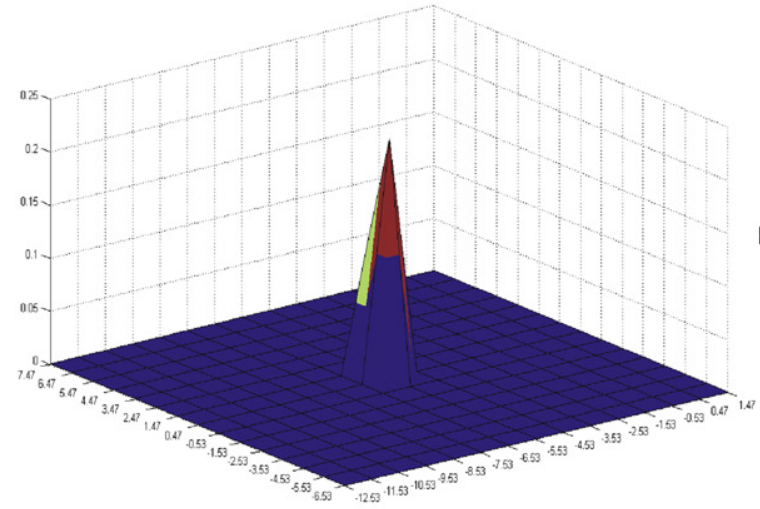
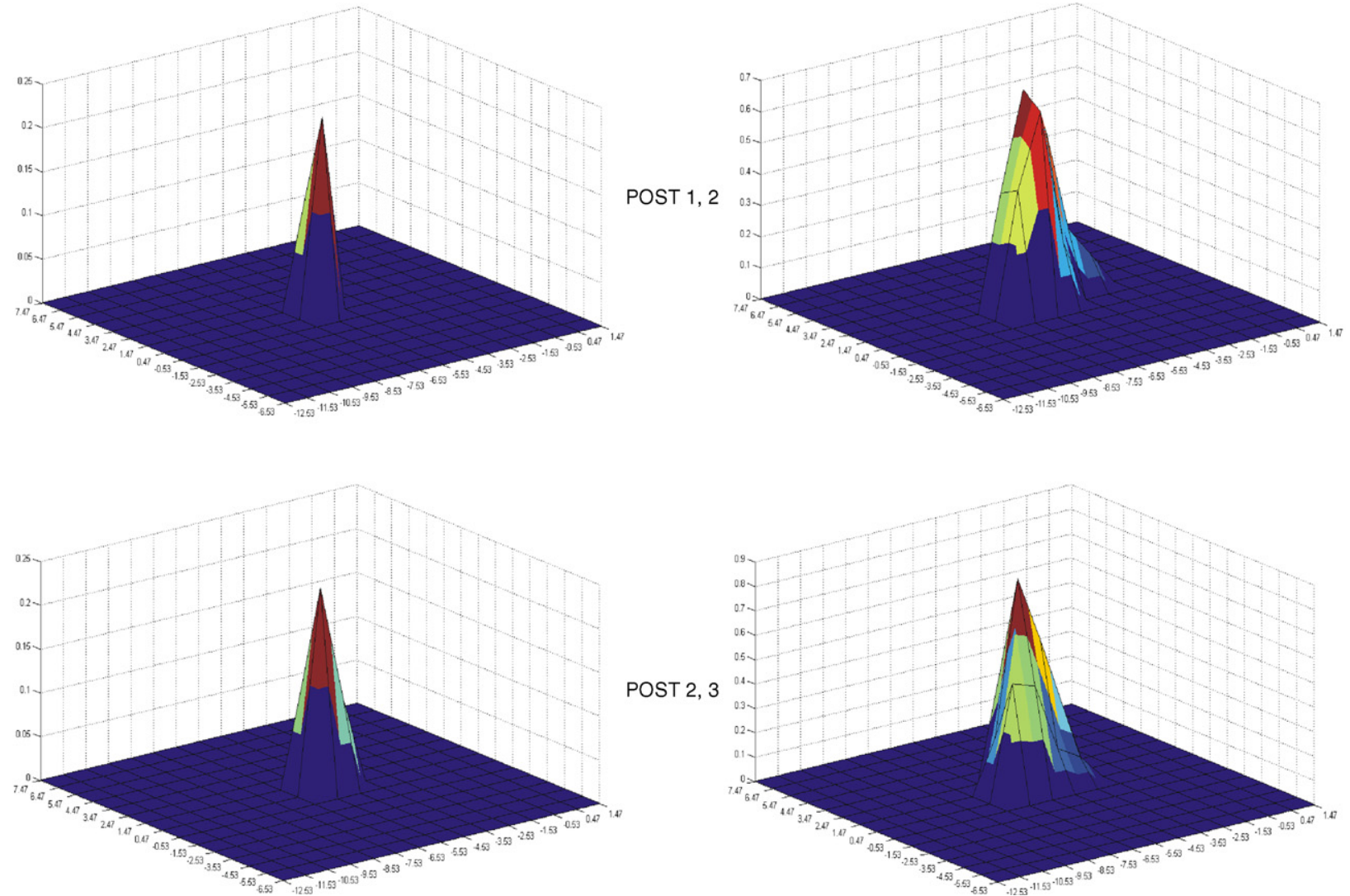

Fig. 3. Three-dimensional plot of the motor cortical representation of the FCR (left column) and the ECR (right column) muscle for a representative subject. The plots depict the mean MEP amplitude (vertical axis) elicited by TMS at each stimulation position before - Pre (top) - and after - Post ${ }^{1,2}$ (middle) - and - Post ${ }^{2,3}$ (bottom) - the intervention. The horizontal axes show the rostrocaudal and mediolateral coordinates of the stimulated scalp sites referenced to the vertex $(0,0)$ in $\mathrm{cm}$. Note the different scaling of the vertical axes for the FCR and ECR. 


\subsection{EMG activity}

Analysis revealed a significant condition effect for FCR EMG activity $[F(2,20)=20.152 ; p<0.05]$. Post hoc testing showed that wrist flexor vibration increased tonic FCR activity significantly, as compared to the pre- and post-vibration levels of EMG activity (pre-vibration: $1.58 \mu \mathrm{V}$, vibration: $2.99 \mu \mathrm{V}$, post-vibration: $1.41 \mu \mathrm{V})(p<0.05)$. With respect to the ECR, a similar condition effect was observed $[F(2,20)=11.888$; $p<0.05]$. Mean EMG activity during vibration was significantly higher than the pre- and post-vibration values (pre-vibration: $1.98 \mu \mathrm{V}$, vibration: $8.09 \mu \mathrm{V}$, post-vibration: $1.78 \mu \mathrm{V})(p<0.05)$. No significant differences between pre- and post-vibration levels of EMG activity were observed, for both the FCR and ECR $(p>0.05)$.

\subsection{Kinesthetic illusion}

Data of one subject were discarded from the analyses since illusion was reported to exceed the physiological joint range of motion for extension (mean $>90^{\circ}$ ). For the remaining 10 subjects, analysis revealed no significant main effect of Time for the experienced angular displacement of the illusionary response $[F(3,27)=1.57, p>0.2]$. Group means were: $26.8^{\circ} \pm 13.0$ (Time 1), $22.5^{\circ} \pm 7.2$ (Time 2), $22.0^{\circ} \pm 6.9$ (Time 3 ) and $20.9^{\circ} \pm 7.8$ (Time 4).

\subsection{Cortico-motor excitability}

Fig. 3 shows three-dimensional plots of the motor cortical representation of the FCR and ECR muscles before (PRE) and after $\left(\mathrm{POST}^{1,2}\right.$ and $\left.\mathrm{POST}^{2,3}\right)$ the intervention. It can be seen that only the corticomotor representation of the ECR muscle changed as a result of the intervention whereas no changes in the map volume and/or area between pre and the two postintervention conditions were noticed for the FCR. Comparing the two muscles (Fig. 4a) by calculating the average of POST ${ }^{1,2}$ and POST $^{2,3}$ subtracted from their corresponding PRE scores shows a substantial increase in the motor cortical representation of ECR $(+11.7 \%$ for MEP amplitude at optimal stimulation position, $+11.9 \%$ for map area and $+26.9 \%$ for map volume) . The FCR mapping variables before and after vibration remained unchanged $(+1.6 \%$ for MEP amplitude, $-10.8 \%$ for map area and $-3.8 \%$ for map volume). A one-way ANOVA with muscle as factor revealed either a significant effect of muscle on map area $[F(1,10)=9.24, p=0.012]$ and volume $[F(1,10)=4.94$, $p=0.050]$ but not on MEP amplitude $[F(1,10)<1]$. These results enabled us to analyze the mapping variables of the ECR separately from those of the FCR and the summary of the values obtained are presented in Table 1.

\subsubsection{MEP amplitude at optimal stimulation position (Fig. 4b)}

One-way ANOVA with Time as the only factor revealed a significant effect of Time on MEP amplitude in the ECR $[F(2,20)=3.69, p=0.043]$. Contrast analysis (Tukey HSD) revealed a sizeable increase with respect to baseline (PRE)
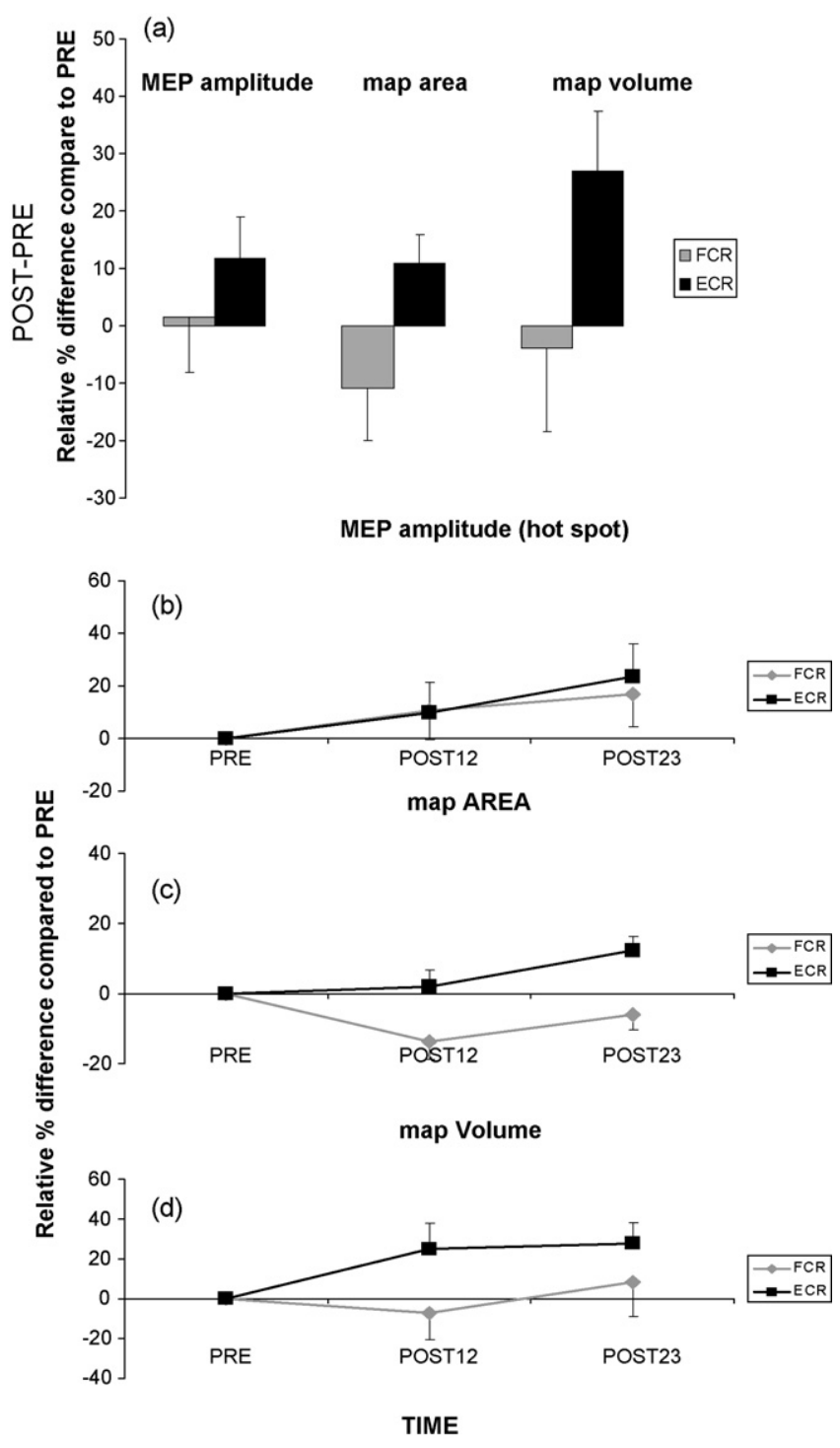

Fig. 4. (a) Mean relative percentage differences between PRE and POST group mean MEP amplitude (at optimal stimulation position), map area and map volume in the right FCR and ECR. (b-d) Time courses of change in MEP amplitude and map area and volume in the same muscles with respect to their baseline levels at the POST ${ }^{12}$ and POST ${ }^{2,3}$ mapping intervals. Data plotted as group mean \pm standard error of the mean (S.E.M.).

amplitude at the $\operatorname{POST}^{2,3}$ interval $(+23.8 \% \pm 6.7$ versus baseline, $p=0.034$ ) but not at the POST ${ }^{1,2}$ interval or between POST $^{1,2}$ and POST ${ }^{2,3}$ intervals (both, $p>0.3$ ). No such effects were observed in the FCR [Time main effect: $F(2,20)<1]$.

\subsubsection{Motor representation area (Fig. $4 c$ )}

No significant differences in the number of active stimulation positions were observed for the FCR muscle following vibration [Time main effect: $F(2,20)=2.15, p>0.1$ ]. With respect to the ECR motor map, a marginally significant increase in map area [Time main effect: $F(2,20)=3.09, p=0.068$ ] emerged following vibration, indicated by the POST $^{2,3}$-PRE comparison $(+12.2 \% \pm 4.2$ versus baseline, $p=0.089)$. Differences in the number of active stimulation positions with respect to baseline at 
Table 1

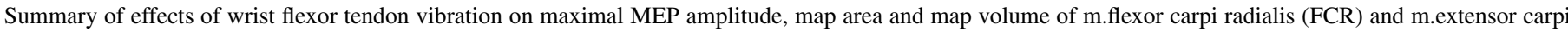
radialis brevis (ECR)

\begin{tabular}{|c|c|c|c|c|}
\hline & PRE & POST & $\mathrm{POST}^{1,2}$ & $\mathrm{POST}^{2,3}$ \\
\hline \multicolumn{5}{|c|}{ MEP amplitude at optimal stimulation position (mV) } \\
\hline FCR & $0.396 \pm 0.234$ & $0.377 \pm 0.207$ & $0.399 \pm 0.199$ & $0.425 \pm 0.220$ \\
\hline ECR & $0.824 \pm 0.238$ & $0.898 \pm 0.229$ & $0.909 \pm 0.326$ & $0.992 \pm 0.273^{*}$ \\
\hline \multicolumn{5}{|c|}{ Map area (number) } \\
\hline FCR & $15.0 \pm 8.3$ & $13.2 \pm 7.7$ & $12.7 \pm 7.6$ & $13.9 \pm 8.4$ \\
\hline ECR & $20.2 \pm 6.8$ & $22.2 \pm 7.0^{\dagger}$ & $20.4 \pm 6.9$ & $22.4 \pm 6.8^{\dagger}$ \\
\hline \multicolumn{5}{|c|}{ Map volume (mV) } \\
\hline FCR & $3.81 \pm 3.80$ & $3.16 \pm 2.45$ & $2.93 \pm 2.22$ & $3.56 \pm 2.84$ \\
\hline ECR & $7.33 \pm 2.85$ & $9.07 \pm 3.97^{\dagger}$ & $8.79 \pm 3.99$ & $9.09 \pm 3.91^{\dagger}$ \\
\hline
\end{tabular}

Data expressed as group mean \pm standard deviation (S.D.).

* $p<0.05$ (compared to the PRE-values).

$\dagger$ Marginally significant differences $p<0.1$ (compared to the PRE-values).

the POST ${ }^{1,2}$ interval or between $\operatorname{POST}^{1,2}$ and $\operatorname{POST}^{2,3}$ intervals were not significant (both, $p>0.1$ ).

\subsubsection{Map volume (Fig. 4c)}

The FCR map volume did not undergo significant changes following the vibration intervention for any the comparisons [Time main effect: $F(2,20)=1.05, p>0.3$ ]. Similar to the ECR map area data, analysis revealed a significant increase in the ECR map volume following the intervention [Time main effect: $F(2,20)=3.54, p=0.048]$. A sizeable increase with respect to baseline (PRE) map volume was observed at the $\mathrm{POST}^{1,2}$ interval $(+24.9 \% \pm 12.6$ versus baseline $)$ and the POST $^{2,3}$ interval $(+27.8 \% \pm 10.5$ versus baseline $)$. However, differences were either statistically marginal (POST ${ }^{2,3}-\mathrm{PRE}, p=0.054$ ) or did not reach significance $\left(\mathrm{POST}^{1,2}-\mathrm{PRE}\right.$ and $\mathrm{POST}^{2,3}-\mathrm{POST}^{1,2}$ : both, $p>0.1)$.

\subsection{Centre of gravity}

Mean ( \pm S.D.) magnitudes of the CoG displacement vectors between pre- and post-vibration for all comparisons were the following for the FCR (PRE-POST: $0.37 \pm 0.25 \mathrm{~cm}$, PRE-POST $^{1,2}: 0.36 \pm 0.20 \mathrm{~cm}$, PRE-POST $\left.^{2,3}: 0.38 \pm 0.25 \mathrm{~cm}\right)$ and ECR (PRE-POST: $0.28 \pm 0.12 \mathrm{~cm}, \quad$ PRE-POST $^{1,2}$ : $0.31 \pm 0.12 \mathrm{~cm}, \quad$ PRE-POST $\left.{ }^{2,3}: 0.29 \pm 0.13 \mathrm{~cm}\right)$. The magnitudes of the CoG shifts were $0.43 \pm 0.33 \mathrm{~cm}$ (FCR) and $0.28 \pm 0.14 \mathrm{~cm}$ (ECR) for the $\mathrm{PRE}^{\mathrm{A}}-\mathrm{PRE}^{\mathrm{B}}$ comparison. Statistical comparison of the displacement vectors across all conditions (the pre-post measures versus the measure obtained within the pre-mapping), revealed no significant differences in the magnitude of the CoG displacement vector $(p>0.05)$.

\section{Discussion}

In the present study, we evaluated the potential capability of artificial activation of the afferent pathways by means of muscle tendon vibration to drive motor cortex reorganization in neurologically intact individuals. For this purpose, we assessed the delayed effects of repetitive sensory stimulation with low- amplitude muscle tendon vibration at $80 \mathrm{~Hz}$ on the expansion of corticomotor representation in the forearm musculature. Extending the observations from previous studies [18,45,46,53-55], we used the activation of Ia muscle spindle afferents as the main source of somatosensory stimulation (even though other sensory receptors may also have been involved). Specifically, the application of $60 \mathrm{~min}$ of wrist flexor tendon vibration elevated the excitability of corticomotor projections to the wrist extensor muscles (ECR) until significant levels of facilitation were obtained over the course of $30 \mathrm{~min}$ post-intervention. In this respect, close similarities were observed between the time course of the increase in corticospinal excitability following our tendon vibration intervention and that obtained after comparable periods of continuous peripheral sensory intervention with electrical stimulation $[15,29,37,41,42]$.

The present intervention evoked a delayed facilitation only in the antagonistic ECR muscle but not in the vibrated FCR muscle. This finding differs from our previous observations in which a similar sensory training protocol through the application of wrist flexor tendon vibration enhanced the excitability of corticospinal pathways of both flexors and extensors, albeit with weaker effects in the FCR than in the ECR [53]. Yet, the present intervention protocol with low vibration amplitude was specifically set to evoke an antagonistic vibratory response (AVR) while at the same time minimizing the emergence of the tonic vibration reflex (TVR) in the FCR. As such, it was expected that the intervention did not induce balanced effects on agonist and antagonist motor circuits in the brain. The latter assumption is consistent with recent work showing that afferent stimulation of specific musculature induces focal effects on corticospinal excitability in targeted muscle(s) without changing the level of motor excitability in general $[45,55]$. It should be noticed though that selective effects of the intervention on corticomotor representation of the wrist muscles may also emerge as a result of discrepancies in the excitability of corticospinal pathways of the flexors and extensors due to, i.e., differences in rest motor threshold and/or size of M1 populations. The latter hypothesis remains to be explored further.

Repetitive stereotyped performance of relatively unskilled movements can evoke plastic changes in M1 movement rep- 
resentations $[4,11,26,36]$. For example, it has been reported that a 15-30 min training period consisting of voluntary repetitive thumb movements, sufficed to establish a rapidly evolving change in the M1 movement representation [11]. Subsequent studies have pointed to long-term potentiation (LTP) as the candidate mechanism underlying this type of 'use-dependent plasticity' [4]. In our experiment, the antagonistic vibratory response (AVR) became apparent in the wrist extensor muscles during vibration. The underlying mechanisms of the AVR are not yet completely understood. However, the most recent findings point to the involvement of a perceptual-to-motor transformation of the vibratory-induced proprioceptive information, most likely occurring at the cortical level rather than being a purely spinal reflex mechanism $[5,6]$. This agrees with the observation that the EMG characteristics of the AVR are correlated with parameters of the experienced kinesthetic illusion during vibration $[5,6]$. Kinesthetic illusions during muscle tendon vibration (i.e., the illusion of the vibrated muscle being stretched) activate contralateral motor cortical areas (M1, SMA) $[31,32,39]$, similar to a closely related experience, i.e., kinesthetic movement imagery [38]. Interestingly, mental motor practice, defined as the conscious representation of performing a movement of a body part without overt action, appears to trigger motor cortex reorganization as powerful as overt motor practice [36]. In summary, besides primarily being a type of somatosensory stimulation, muscle tendon vibration elicits a kinesthetic illusion and its associated AVR. The repetitive experience of both phenomena contains large similarities with two procedures, which are known to induce cortical reorganization changes, i.e., repetitive overt practice of relatively unskilled movements and repetitive mental practice. As such, we hypothesized that sensory 'illusion' induced by muscle tendon vibration is an amplifying factor in driving corticomotor excitability changes.

The major finding of the present study was that a clear tendency to increase the motor output area as well as the motor map volume became apparent following interventional muscle tendon vibration. An important issue to address is to what extent the functional M1 representation obtained by TMS mapping corresponds with the anatomical M1 representation of the target muscle. Evidently, the TMS map is only indirectly related to the origin and distribution of the underlying corticomotor projection. Moreover, the spatial location and extent of the map is dependent on factors, such as coil orientation [66,67], current spread [49], coil distance [58] and the excitability of the underlying corticospinal projection [40]. Nevertheless, TMS maps agree well with the location of the hand area within M1, as evidenced by other imaging modalities [62,23]. Moreover, the technique has demonstrated a remarkable capability to detect shifts in map position with a high resolution [57]. A second issue is the identification of the required boundary conditions to ascribe map changes to underlying neuroplastic alterations. As such, changes in map area per se cannot be assumed to be due solely due to a true reorganization, but might reflect a change in the level of excitability of a topographically fixed projection $[40,50]$. For this reason, changes in measures of map location rather than area have been shown to provide a better indication of reorganization of the underlying cortical representation $[50,58]$. In the present study, there was no significant displacement of the CoGs following interventional muscle tendon vibration. It can be suggested that the observed increase in the motor representations of the wrist musculature was mediated by increasing pre-synaptic axonal excitability and/or by generating a lasting increase in excitatory synaptic activity $[3,48,50]$. As such, the intervention with tendon vibration in our study may have influenced the excitability of topographically fixed projections without changing the actual locus of the hand representation in M1. Alternatively, the current intervention may have equally increased the excitability of the cortical motor neuron populations in such a way that there was no evident shift in the CoG's of the cortical map.

The fact that the effects obtained in our study outlasted the period of kinaesthetic stimulation by more than $30 \mathrm{~min}$, opens perspectives for therapeutic manipulation of brain plasticity [e.g. $13,15,41,42]$. Consequently, an important future goal is to investigate to what extent excitability and reorganization changes following somatosensory stimulation are related to functional improvements in movement control. Up to now, no significant changes in motor performance have been reported following interventional peripheral nerve stimulation or muscle tendon vibration in healthy humans. However, other studies $[13,15]$ reported significant improvements in swallowing function and pinch strength, outlasting the period of pharyngeal and median nerve stimulation, respectively. Therefore, it might be that substantial deficits in motor performance after brain injury are required to measure behavioural gains following interventional stimulation paradigms. Moreover, the possibility to 'tune' M1 plastic changes by means of manipulating the parameters of somatosensory stimulation [68], awaits the development of optimized intervention techniques.

\section{Acknowledgement}

Support for this study was provided through a grant from the Research Programme of the Research Foundation-Flanders (FWO, \#G.0292.05).

\section{References}

[1] Brasil-Neto JP, Cohen LG, Pascual-Leone A, Jabir FK, Wall RT, Hallett M. Rapid reversible modulation of human motor outputs after transient deafferentation of the forearm: a study with transcranial magnetic stimulation. Neurology 1992;42:1302-6.

[2] Brasil-Neto JP, Valls-Sole J, Pascual-Leone A, Cammarota A, Amassian VE, Cracco R, et al. Rapid modulation of human cortical motor outputs following ischaemic nerve block. Brain 1993;116:511-25.

[3] Buonomano DV, Merzenich MM. Cortical plasticity: from synapses to maps. Ann Rev Neurosci 1998;21:149-86.

[4] Bütefisch CM, Davis BC, Wise SP, Sawaki L, Kopylev L, Classen J, et al. Mechanisms of use-dependent plasticity in the human motor cortex. PNAS 2000;97:3661-5.

[5] Calvin-Figuière S, Romaiguere P, Gilhodes JC, Roll JP. Antagonist motor responses correlate with kinesthetic illusions induced by tendon vibration. Exp Brain Res 1999;124:342-50.

[6] Calvin-Figuière S, Romaiguere P, Roll JP. Relations between the directions of vibration-induced kinesthetic illusions and the pattern of activation of antagonist muscles. Brain Res 2000;881:128-38. 
[7] Carel C, Loubinoux I, Boulanouar K, Manelfe C, Rascol O, Celsis P, et al. Neural substrate for the effects of passive training on sensorimotor cortical representation: a study with functional magnetic resonance imaging in healthy subjects. J Cereb Blood Flow Metab 2000;20:478-84.

[8] Charlton CS, Ridding MC, Thompson PD, Miles TS. Prolonged peripheral nerve stimulation induces persistent changes in excitability of human motor cortex. J Neurol Sci 2003;208:79-85.

[9] Chen R, Corwell B, Yaseen Z, Hallett M, Cohen LG. Mechanisms of cortical reorganization in lower-limb amputees. J Neurosci 1998;18:344350.

[10] Cicinelli P, Traversa R, Rossini PM. Post-stroke reorganization of brain motor output to the hand: a 2-4 month follow-up with focal magnetic transcranial stimulation. Electroencephalogr Clin Neurophysiol 1997; 105:438-50.

[11] Classen J, Liepert J, Wise SP, Hallett M, Cohen LG. Rapid plasticity of human cortical movement representation induced by practice. J Neurophysiol 1998;79:1117-23.

[12] Cohen LG, Bandinelli S, Findley TW, Hallett M. Motor reorganization after upper limb amputation in man. A study with focal magnetic stimulation. Brain 1991;114:615-27

[13] Conforto AB, Kaelin-Lang A, Cohen LG. Increase in hand muscle strength of stroke patients after somatosensory stimulation. Ann Neurol 2002;51:122-5.

[14] Donoghue JP. Plasticity of adult sensorimotor representations. Curr Opin Neurobiol 1995;5:749-54.

[15] Fraser C, Power M, Hamdy S, Rothwell J, Hobday D, Hollander I, et al. Driving plasticity in human adult motor cortex is associated with improved motor function after brain injury. Neuron 2002;34:831-40.

[16] Fuhr P, Cohen LG, Dang N, Findley TW, Haghighi S, Oro J, et al. Physiological analysis of motor reorganization following lower limb amputation. Electroencephalogr Clin Neurophysiol 1992;85:53-60.

[17] Goodwin GM, McCloskey DI, Matthews PB. Proprioceptive illusions induced by muscle vibration: contribution by muscle spindles to perception? Science 1972;175:1382-4

[18] Hamdy S, Rothwell JC, Aziz Q, Singh KD, Thompson DG. Long-term reorganization of human motor cortex driven by short-term sensory stimulation. Nat Neurosci 1998;1:64-8.

[19] Hund-Georgiadis M, von Cramon DY. Motor-learning-related changes in piano players and non-musicians revealed by functional magneticresonance signals. Exp Brain Res 1999;125:417-25.

[20] Karni A, Meyer G, Jezzard P, Adams MM, Turner R, Ungerleider LG. Functional MRI evidence for adult motor cortex plasticity during motor skill learning. Nature 1995;377:155-8.

[21] Karni A, Meyer G, Rey-Hipolito C, Jezzard P, Adams MM, Turner R, et al. The acquisition of skilled motor performance: fast and slow experiencedriven changes in primary motor cortex. Proc Natl Acad Sci 1998;95:861-8.

[22] Kossev A, Siggelkow S, Schubert M, Wohlfarth K, Dengler R. Muscle vibration: different effects on transcranial magnetic and electrical stimulation. Muscle Nerve 1999;22:946-8.

[23] Krings T, Buchbinder BR, Butler WE, Chiappa KH, Jiang HJ, Cosgrove GR, et al. Functional magnetic resonance imaging and transcranial magnetic stimulation: complementary approaches in the evaluation of cortical motor function. Neurology 1997;48:1406-16.

[24] Levy Jr WJ, Amassian VE, Traad M, Cadwell J. Focal magnetic coil stimulation reveals motor cortical system reorganized in humans after traumatic quadriplegia. Brain Res 1990;510:130-4.

[25] Liepert J, Tegenthoff M, Malin JP. Changes of cortical motor area size during immobilization. Electroencephalogr Clin Neurophysiol 1995;97:382-6.

[26] Liepert J, Terborg C, Weiller C. Motor plasticity induced by synchronized thumb and foot movements. Exp Brain Res 1999;125:435-9.

[27] Liepert J, Bauder H, Wolfgang HR, Miltner WH, Taub E, Weiller C. Treatment-induced cortical reorganization after stroke in humans. Stroke 2000;31:1210-6.

[28] Lotze M, Kaethner RJ, Erb M, Cohen LG, Grodd W, Topka H. Comparison of representational maps using functional magnetic resonance imaging and transcranial magnetic stimulation. Clin Neurophysiol 2003;114:30612 .
[29] McKay DR, Ridding MC, Thompson PD, Miles TS. Induction of persistent changes in the organisation of the human motor cortex. Exp Brain Res 2002; 143:342-9.

[30] Mortifee P, Stewart H, Schulzer M, Eisen A. Reliability of transcranial magnetic stimulation for mapping the human motor cortex. Electroencephalogr Clin Neurophysiol 1994;93:131-7.

[31] Naito E, Ehrsson HH, Geyer S, Zilles K, Roland PE. Illusory arm movements activate cortical motor areas: a positron emission tomography study. J Neurosci 1999; 19:6134-44.

[32] Naito E, Ehrsson HH. Kinesthetic illusion of wrist movement activates motor-related areas. Neuroreport 2001;12:3805-9.

[33] Oldfield RC. The assessment and analysis of handedness: the Edinburgh inventory. Neuropsychologia 1971;9:97-113.

[34] Pascual-Leone A, Cammarota A, Wassermann EM, Brasil-Neto JP, Cohen LG, Hallett M. Modulation of motor cortical outputs to the reading hand of braille readers. Ann Neurol 1993;34:33-7.

[35] Pascual-Leone A, Grafman J, Hallett M. Modulation of cortical motor output maps during development of implicit and explicit knowledge. Science 1994;263:1287-9.

[36] Pascual-Leone A, Nguyet D, Cohen LG, Brasil-Neto JP, Cammarota A, Hallett M. Modulation of muscle responses evoked by transcranial magnetic stimulation during the acquisition of new fine motor skills. J Neurophysiol 1995;74:1037-45.

[37] Pitcher JB, Ridding MC, Miles TS. Frequency-dependent, bi-directional plasticity in motor cortex of human adults. Clin Neurophysiol 2003;114:1265-71.

[38] Porro CA, Francescato MP, Cettolo V, Diamond ME, Baraldi P, Zuiani $\mathrm{C}$, et al. Primary motor and sensory cortex activation during motor performance and motor imagery: a functional magnetic resonance imaging study. J Neurosci 1996;16:7688-98.

[39] Radovanovic S, Korotkov A, Ljubisavljevic M, Lyskov E, Thunberg J, Kataeva $\mathrm{G}$, et al. Comparison of brain activity during different types of proprioceptive inputs: a positron emission tomography study. Exp Brain Res 2002;143:276-85.

[40] Ridding MC, Rothwell JC. Stimulus/response curves as a method of measuring motor cortical excitability in man. Electroencephalogr Clin Neurophysiol 1997;105:340-4.

[41] Ridding MC, Brouwer B, Miles TS, Pitcher JB, Thompson PD. Changes in muscle responses to stimulation of the motor cortex induced by peripheral nerve stimulation in human subjects. Exp Brain Res 2000;131:13543.

[42] Ridding MC, McKay DR, Thompson PD, Miles TS. Changes in corticomotor representations induced by prolonged peripheral nerve stimulation in humans. Clin Neurophysiol 2001;112:1461-9.

[43] Roll JP, Vedel JP. Kinaesthetic role of muscle afferents in man, studied by tendon vibration and microneurography. Exp Brain Res 1982;47:177-90.

[44] Roll JP, Vedel JP, Ribot E. Alteration of proprioceptive messages induced by tendon vibration in man: a microneurographic study. Exp Brain Res 1989;76:213-22.

[45] Rosenkranz K, Rothwell JC. Differential effect of muscle vibration on intracortical inhibitory circuits in humans. J Physiol 2003;551:649 60.

[46] Rosenkranz K, Rothwell JC. Differences between the effects of three plasticity inducing protocols on the organization of the human motor cortex. Eur J Neurosci 2006;23(3):822-9.

[47] Rossini PM, Barker AT, Berardelli A, Caramia MD, Caruso G, Cracco RQ et al. Non-invasive electrical and magnetic stimulation of the brain, spinal cord and roots: basic principles and procedures for routine clinical application. Report of an IFCN committee. Electroencephalogr Clin Neurophysiol 1994;91:79-92.

[48] Rossini PM, Rossi S, Tecchio F, Pasqualetti P, Finazzi-Agro A, Sabato A Focal brain stimulation in healthy humans: motor maps changes following partial hand sensory deprivation. Neurosci Lett 1996;214:191-5.

[49] Roth BJ, Saypol JM, Hallett M, Cohen LG. A theoretical calculation of the electric field induced in the cortex during magnetic stimulation. Electroencephalogr Clin Neurophysiol 1991;81:47-56.

[50] Siebner HR, Rothwell J. Transcranial magnetic stimulation: new insights into representational cortical plasticity. Exp Brain Res 2003;148:1-16. 
[51] Stefan K, Kunesch E, Cohen LG, Benecke R, Classen J. Induction of plasticity in the human motor cortex by paired associative stimulation. Brain 2000;123:572-84.

[52] Stefan K, Kunesch E, Benecke R, Cohen LG, Classen J. Mechanisms of enhancement of human motor cortex excitability induced by interventional paired associative stimulation. J Physiol 2002;543:699-708.

[53] Steyvers M, Levin O, Van Baelen M, Swinnen SP. Corticospinal excitability changes following prolonged muscle tendon vibration. Neuroreport 2003;14:1901-5.

[54] Steyvers M, Levin O, Verschueren SM, Swinnen SP. Frequency-dependent effects of muscle tendon vibration on corticospinal excitability: a TMS study. Exp Brain Res 2003;151:9-14.

[55] Swayne O, Rothwell J, Rosenkranz K. Transcallosal sensorimotor integration: effects of sensory input on cortical projections to the contralateral hand. Clin Neurophysiol 2006;117(4):855-63.

[56] Thickbroom GW, Byrnes ML, Mastaglia FL. A model of the effect of MEP amplitude variation on the accuracy of TMS mapping. Clin Neurophysiol 1999;110:941-3.

[57] Thickbroom GW, Byrnes ML, Mastaglia FL. Methodology and application of TMS mapping. Electroencephalogr Clin Neurophysiol 1999;51:4854.

[58] Thickbroom GW, Mastaglia FL. Mapping studies. In: Pascual Leone A, et al., editors. Handbook of transcranial magnetic stimulation. London, UK: Arnold Publishers; 2002.

[59] Topka H, Cohen LG, Cole RA, Hallett M. Reorganization of corticospinal pathways following spinal cord injury. Neurology 1991;41:1276-83.

[60] Traversa R, Cicinelli P, Bassi A, Rossini PM, Bernardi G. Mapping of motor cortical reorganization after stroke. A brain stimulation study with focal magnetic pulses. Stroke 1997;28:110-7.
[61] Wassermann EM, McShane LM, Hallett M, Cohen LG. Noninvasive mapping of muscle representations in human motor cortex. Electroencephalogr Clin Neurophysiol 1992;85:1-8.

[62] Wassermann EM, Wang B, Zeffiro TA, Sadato N, Pascual-Leone A, Toro $\mathrm{C}$, et al. Locating the motor cortex on the MRI with transcranial magnetic stimulation and PET. Neuroimage 1996;3:1-9.

[63] Wassermann EM. Risk and safety of repetitive transcranial magnetic stimulation: report and suggested guidelines from the International Workshop on the Safety of Repetitive Transcranial Magnetic Stimulation, June 5-7, 1996. Electroencephalogr Clin Neurophysiol 1998;108:1-16.

[64] Weiller C, Juptner M, Fellows S, Rijntjes M, Leonhardt G, Kiebel S, et al. Brain representation of active and passive movements. Neuroimage 1996;4:105-10.

[65] Werhahn KJ, Fong JK, Meyer BU, Priori A, Rothwell JC, Day BL, et al. The effect of magnetic coil orientation on the latency of surface EMG and single motor unit responses in the first dorsal interosseous muscle. Electroencephalogr Clin Neurophysiol 1994;93:138-46.

[66] Wilson SA, Thickbroom GW, Mastaglia FL. Transcranial magnetic stimulation mapping of the motor cortex in normal subjects. The representation of two intrinsic hand muscles. J Neurol Sci 1993;118:134-44.

[67] Wilson SA, Day BL, Thickbroom GW, Mastaglia FL. Spatial differences in the sites of direct and indirect activation of corticospinal neurones by magnetic stimulation. Electroencephalogr Clin Neurophysiol 1996;101:255-61.

[68] Wolters A, Sandbrink F, Schlottmann A, Kunesch E, Stefan K, Cohen LG, et al. A temporally asymmetric Hebbian rule governing plasticity in the human motor cortex. J Neurophysiol 2003;89:2339-45.

[69] Ziemann U, Hallett M, Cohen LG. Mechanisms of deafferentation-induced plasticity in human motor cortex. J Neurosci 1998;18:7000-7. 